Catherine BAROIN*

\title{
La circulation et les droits sur le bétail, clés de la vie sociale chez les Toubou (Tchad, Niger)
}

\section{Résumé}

Chez les pasteurs toubou la circulation du bétail entre les familles, qui dépend en large partie de leur système matrimonial, apparaît comme la clé de voûte de l'organisation sociale puisqu'elle détermine les droits sur les animaux comme le statut des personnes. La comparaison avec d'autres sociétés pastorales acéphales est esquissée en conclusion.

\section{Mots clefs}

Toubou, Tchad, Niger, transferts de bétail, droits sur le bétail, sociétés pastorales acéphales

\section{Circulation and rights on cattle}

keys for social life in the Tubu (Tchad, Niger)

\section{Abstract}

Among Tubu pastoralists animal transfers, which derive mainly from the marriage system, play a basic role in the social structure since they determine individual rights on the animals as well as personal status. Tubu society is compared to other african acephalous pastoral societies in conclusion.

Keywords

Tubu, Chad, Niger, animal transfers, rights on animals, acephalous pastoral societies

\footnotetext{
${ }^{*}$ CNRS, UMR 7041
} 
Comme l'écrit Bonfiglioli, "les problèmes relatifs à la propriété animale dans les sociétés pastorales ont été très peu étudiés, contrairement aux problèmes de propriété foncière des systèmes agraires" (1988 : 184). Or lorsque les animaux sont la principale, voire l'unique richesse, les droits sur le bétail sont tout aussi cruciaux que ceux d'accès à la terre pour les sociétés agricoles. C'est donc ce thème que nous aborderons ici, à propos d'une société pastorale saharo-sahélienne, celle des Toubou.

Les Toubou sont un peuple d'éleveurs nomades qui occupe un quart du Sahara, entre le lac Tchad et la Libye. Ils se subdivisent en deux vastes groupes apparentés, aux contours flous car les intermariages sont fréquents. Ce sont les Teda au nord et les Daza au sud. Les uns et les autres parlent deux dialectes d'une même langue nilo-saharienne. Les Teda en tant que sahariens sont plutôt éleveurs de chameaux ${ }^{1}$, tandis que les Daza associent à cet élevage, en zone sahélienne, celui des bovins. Du petit bétail s'y ajoute fréquemment dans les deux cas. Ces pasteurs sont les voisins orientaux des Touareg, dont ils sont séparés par un espace inoccupé, le désert du Ténéré, au Niger. Comme ils vivent sous les mêmes latitudes, les Touareg et les Toubou occupent un environnement naturel assez semblable. Les pluies, très aléatoires, sont insuffisantes pour cultiver, et le seul mode d'exploitation possible de ces vastes espaces est l'élevage extensif d'un bétail adapté. De ce fait, ces nomades partagent à bien des égards le même mode de vie et font le même usage des troupeaux. Ils privilégient l'élevage de femelles (vaches et chamelles) parce qu'ils consomment le lait, frais ou caillé, tandis que ces bêtes assurent l'objectif prioritaire des éleveurs, qui est le croît du troupeau. Ces similitudes s'étendent d'ailleurs aux autres pasteurs du Sahara, qui pour la plupart pratiquent le même type d'élevage.

Plutôt qu'à l'usage du bétail, c'est donc aux droits sur les animaux que nous nous intéresserons dans cet article, notre objectif étant de montrer qu'ils sont une clé essentielle pour comprendre la nature des rapports sociaux chez ces pasteurs. Ces droits, en effet, résultent de réseaux d'échanges entre individus, dont l'analyse permet de saisir l'architecture logique de la société toubou dans tous ses principaux aspects. Nous verrons ainsi comment ces échanges fondent l'économie interne de cette société pastorale, comment les droits qui en découlent éclairent non seulement la nature des rapports interindividuels tant au sein de la parenté qu'en dehors, mais aussi le statut de chacun et la logique d'ensemble de l'organisation sociale et politique. Nous montrerons également que, sur tous ces points, les Toubou sont foncièrement différents des autres pasteurs nomades africains.

\footnotetext{
${ }^{1}$ On parle en Afrique de chameaux, mais ces animaux n'ont qu'une bosse et sont en fait des dromadaires.
} 
Ce sont donc les causes de l'originalité culturelle de ce peuple que nous pourrons ainsi dégager, en concluant cette étude par quelques comparaisons.

\section{DES FAMILLES RESTREINTES AUTONOMES, A LA MARGE DE L'ECONOMIE DE MARCHE}

Soulignons d'abord qu'encore de nos jours, la vie économique des Toubou, pour sa plus grande part, se situe en dehors de l'économie de marché. Les marchés sont loin des campements et l'on s'y rend le moins possible. D'ailleurs, ce n'est pas par l'achat qu'un Toubou acquiert d'habitude ses animaux, comme nous le verrons plus bas. L'usage de l'argent, dans les campements, n'a pas cours et rien n'y est à vendre. C'est seulement quand il faut payer l'impôt, ou acheter quelques denrées indispensables, qu'un éleveur se rend au marché pour y vendre une ou deux bêtes ${ }^{2}$. Il en vend le moins possible, juste ce qu'il faut pour ses achats, et choisit de préférence un mâle (taureau ou chameau) ou une femelle stérile, pour ne pas hypothéquer le croît de son troupeau. L'argent de la vente, en dehors de l'impôt, est aussitôt dépensé en totalité pour acquérir quelques produits qui sont rapportés au campement. Pour l'essentiel il s'agit, de temps à autre, d'un sac de mil, d'un peu de thé et de sucre, d'un vêtement ou d'un récipient. Le thé, bu très sucré, est d'autant plus apprécié qu'il est la boisson sociale par excellence, tandis que le mil constitue, avec le lait, la base de la nourriture. Pourtant lorsque le sac de mil familial est vide, il peut se passer plusieurs semaines avant qu'on ne parte s'approvisionner à nouveau. Le manque de mil n'est pas rare, et la retenue devant les aliments est un comportement caractéristique appris dès le plus jeune âge (Baroin, 2005). Il importe en effet de limiter au maximum les ventes d'animaux, afin de préserver le capital dont vit chaque famille. Sauf exception, on ne consomme donc pas le gros bétail. C'est seulement pour une grande cérémonie, mariage ou funérailles, que l'on tue un bovin, voire deux. Pour les occasions plus modestes que sont une naissance ou une circoncision, c'est une chèvre que l'on abat. Ces animaux élevés en petit nombre constituent en effet une réserve de viande sur pieds.

La famille restreinte forme une unité économique autonome. Abritée par une tente de nattes qui appartient à l'épouse, elle exploite et conduit où bon lui semble son troupeau. La résidence, libre, est infléchie toutefois par les affinités et les liens de parenté. La tente en général est montée au voisinage de quelques autres, souvent occupées par des parents proches, à proximité d'un puits. Mais les configurations résidentielles fluctuent, au gré

\footnotetext{
${ }^{2}$ Les dépenses d'éducation et de santé sont négligeables, car les infrastructures scolaires et sanitaires sont presque inexistantes en pays toubou.
} 
des stratégies dont décide chaque chef de famille et qu'impose la recherche de pâturage.

Cette liberté formelle n'exclut pas cependant de multiples obligations qui lient entre elles les familles apparentées. Pour comprendre à la fois ce qui fonde l'indépendance de chaque famille et la nature de ces obligations, il faut d'abord retracer la genèse de la cellule familiale toubou. Elle est le fruit d'un processus qui, dans les grandes lignes, se répète dans chaque cas à l'identique. Nous exposerons donc comment se constitue une famille nucléaire, c'est-à-dire comment s'apparient au départ les partenaires conjugaux, et ensuite d'où provient le troupeau dont ils tireront leur subsistance. C'est après la description de ce processus que nous pourrons procéder à l'analyse de ses répercussions juridiques et sociales. Nous examinerons dans un premier temps les droits sur le bétail qui en découlent et la manière dont ils influent sur les rapports sociaux au niveau individuel. Puis, dans un second temps, nous étudierons les conséquences du mode de constitution des familles sur la vie économique, sociale et politique de cette société pastorale dans son ensemble ${ }^{3}$.

\section{LE SYSTEME MATRIMONIAL}

Le premier facteur à prendre en compte est la règle de mariage. Or sur ce point, les Toubou diffèrent de toutes les autres sociétés pastorales voisines. Tandis que chez les Touaregs, les Peuls ou les Arabes, le mariage entre proches parents est admis et même recherché, c'est strictement l'inverse chez les Toubou. Pour eux, la prohibition de ce type d'union est très large puisque le mariage est interdit, disent-ils, «lorsqu'il y a trois grands-pères », c'est-à-dire un trisaïeul commun. A cet égard, la parenté par les femmes compte autant que la parenté par les hommes, et une arrièrearrière-grand-mère commune constitue un empêchement au mariage au même titre qu'un ancêtre masculin. Cette règle est largement respectée, et même l'influence grandissante du modèle culturel islamo-arabique, auprès de ces pasteurs qui sont musulmans comme tous les Sahariens, ne les en a pas détournés. En conséquence, il est nécessaire de contracter chaque nouvelle alliance loin en dehors du cercle des proches parents. Ceci contribue fortement au brassage de la population qui caractérise le monde

\footnotetext{
${ }^{3}$ Les données qui suivent sont le fruit d'enquêtes menées pour l'essentiel chez les Toubou de l'Est du Niger, entre 1969 et 1972. Elles n'ont malheureusement pas été réactualisées par de nouvelles recherches, en raison de problèmes politiques et d'accessibilité du terrain. Cependant le système social mis en évidence à leur propos (Baroin, 1985) s'observe également, dans une très large mesure, chez les Toubou du Tchad, comme des travaux comparatifs (Baroin, 1988) et des enquêtes au Borkou (1990-1991) ont permis de l'établir (Baroin, 1994).
} 
toubou : les parents de chacun sont disséminés dans un très grand nombre de campements, souvent très éloignés les uns des autres.

Les jeunes filles sont presque toutes mariées avant l'âge de 20 ans, tandis que les garçons le sont en général une dizaine d'années plus tard. Les parents d'une jeune fille attendent les offres d'un prétendant, tandis que ceux d'un garçon recherchent activement pour lui un parti convenable, c'est-à-dire une fiancée dont les parents sont, autant que possible, riches et nombreux. Ces considérations, comme nous le verrons, sont en effet primordiales pour l'avenir du couple. Une fois le choix arrêté et l'accord obtenu pour ce premier mariage, le jeune homme doit verser à son futur beau-père un "prix de la fiancée » benõ, dont le montant fait lui aussi l'objet d'un accord préalable entre les deux familles. Cette prestation peut varier du simple au triple, en fonction de la richesse des partenaires en cause. Mais il s'agit toujours, pour une jeune fille, d'un versement considérable, que le garçon est tenu de payer intégralement avant que le mariage n'ait lieu, au contraire de nombreuses autres sociétés où le mariage instaure une dette qui se paye ensuite, tout au long de la vie.

Ce versement est évalué en "pièces d'étoffe" sande, pl. sanda. Il s'agit d'une unité de compte héritée du passé, et assez générale en Afrique ${ }^{4}$. Mais c'est une unité de compte uniquement, car ces pièces d'étoffe ne s'observent nulle part dans les campements toubou, on y fait simplement référence pour évaluer diverses prestations. La nature des paiements de mariage a varié au fil du temps. Les versements en thé et sucre se sont développés vers 1940-1950, période où la consommation de thé chez les Toubou connaissait un succès grandissant. Puis, vers 1970, les paiements sous forme d'animaux ont pris le pas sur le thé et le sucre, selon le désir du père de la future mariée car c'est lui qui choisit la forme sous laquelle ce versement doit lui être remis. Alors, ce sont par exemple dix chamelles adultes que le jeune homme doit remettre à son futur beau-père.

En règle habituelle le jeune célibataire ne possède pas un tel nombre d'animaux. Il peut avoir reçu à la naissance une ou deux têtes de bétail d'un parent ou d'une parente proche, comme c'est d'usage pour tout bébé, garçon ou fille. Ce cadeau s'appelle cicukti, terme formé à partir du verbe « embrasser» cucukti. Ensuite vient pour un garçon, vers 13 ans, une nouvelle occasion de recevoir des cadeaux: la circoncision ${ }^{5}$. Chaque circoncis reçoit alors au moins 3 ou 4 animaux de divers parents et parentes, parfois davantage. Ce bétail est appelé kèlèktè, terme formé à partir du mot

\footnotetext{
${ }^{4}$ Par exemple, J. Swift indique qu'en Somalie, au XIX ${ }^{\mathrm{e}}$ siècle, "cloth was used as a general standard in which the value of most goods was assessed" (1979:450).

${ }^{5}$ Les filles n'ont aucune occasion équivalente de recevoir des cadeaux; elles ne sont pas excisées.
} 
kulum, qui désigne la « clochette des circoncis ». En effet, c'est en agitant cette clochette suspendue à un bâton que le jeune circoncis fait le tour de ses parents pour solliciter ces dons. Mais les animaux qu'il reçoit, comme celui ou ceux qui lui avaient été donnés à sa naissance, intègrent le troupeau de son père. Ce dernier en dispose selon sa volonté, comme c'est le cas de tout bétail offert à ses enfants non mariés.

Quand il arrive en âge de se marier, le jeune homme ne récupère pas pour autant le bétail qui lui a été remis et dont son père a la gestion. Ce dernier peut lui donner quelques animaux, mais leur nombre est insuffisant pour payer le "prix de la fiancée ». D'ailleurs si le père en donnait davantage, c'est l'avenir de son propre foyer et le mariage de ses autres enfants encore jeunes qu'il risquerait de compromettre. Le futur marié doit donc trouver ailleurs le bétail requis. Pour le rassembler, il part en tournée afin de solliciter ses parents et parentes, paternels et maternels, qui sont disséminés dans divers campements parfois très distants.

Chacun de ces parents, au bout d'un temps de visite plus ou moins long, lui fait le cadeau qu'il est venu chercher. Ce don est dénommé troko pl. troka, terme qui désigne aussi l'aide exceptionnelle qu'on apporte à un parent dans le besoin, par exemple pour l'aider à payer des frais d'hôpital. La personne sollicitée ne peut refuser, car ce serait un déshonneur que de ne pas venir en aide à un jeune parent qui cherche à se marier. Cette obligation est tout aussi impérative que celle qui pèse, en cas de meurtre, sur le parent d'un meurtrier : il lui est impossible de refuser de contribuer au «prix du sang », compensation versée à la famille de la victime pour lever la menace de vengeance. Il y a donc obligation de donner, et pas n'importe quoi. Ces aides au mariage ne peuvent être que d'une seule nature. Ce sont nécessairement des têtes de gros bétail (bovins ou chameaux), une au minimum, car il serait méprisable de faire un cadeau de moindre valeur. Le nombre de parents sollicités par un futur candidat au mariage et l'importance de chacun des dons qu'il reçoit varient d'un cas à l'autre. Ainsi lors d'une enquête effectuée sur 8 mariages chez les Toubou de l'Est du Niger, en 1972, le nombre de donneurs variait de 3 à 25 personnes, avec une moyenne de 13 donneurs par mariage, et le nombre d'animaux reçus par le futur marié allait de 10 à 25 bêtes (Baroin $1985: 215 s s q$ ).

Il est clair que le nombre et la richesse des membres de la parenté du fiancé influent sur le déroulement des opérations, de même que l'éloignement des campements où ils résident et leur promptitude à se montrer généreux. Aussi le jeune homme qui entreprend cette campagne met-il un temps plus ou moins long pour obtenir l'ensemble des animaux qu'il lui faut. Cette démarche peut prendre deux ans ou davantage, et même parfois jusqu'à dix ans. Le risque est alors que la jeune fille soit donnée en 
mariage à un rival plus rapide et plus fortuné car tant que le «prix de la fiancée » n'est pas intégralement versé, la famille de la future mariée n'est tenue par aucun engagement. Pour contrer cette menace, ou par simple désir de montrer leur bravoure, beaucoup de futurs maris enlèvent leur promise avant d'avoir tout payé. Mais le rapt est un acte codifié. Il ne peut avoir lieu sans la complicité d'un parent ou d'une parente de la jeune fille. Le mariage par rapt honore une femme et témoigne de l'audace de son conjoint. Mais il ne le dispense nullement de payer ensuite ce qu'il doit encore à son beaupère. Le mariage sinon serait invalidé, et les parents de l'épouse en droit d'exiger le retour de leur fille.

Dans la procédure normale, à mesure que le jeune homme reçoit des animaux de ses divers parents, il effectue des versements successifs à son futur beau-père, sous la forme demandée par celui-ci. Il lui donne les dix chamelles requises si tel est le cas. Ou bien, s'il préfère du thé et du sucre, le futur gendre va vendre au marché le bétail qui lui a été donné, vaches, chameaux, veaux et chamelons, reçus de parents plus ou moins nombreux, pour en rapporter du thé et du sucre.

Le cycle des transferts, toutefois, ne s'arrête pas là. Le beau-père se réserve une part des biens reçus de son futur gendre, mais il en redistribue la majeure partie à ses propres parents et, dans une moindre mesure, à des parents de sa femme. Ce sont donc les parents paternels et maternels de la fiancée qui bénéficient de ces dons, dénommés tewa. Le montant reçu par chacun ou chacune se monte en principe à 7 unités de compte (les "pièces de percale »sanda), soit l'équivalent d'une chamelle de trois ans. Il peut être supérieur (le maximum observé en 1972 dans l'Est du Niger était de 20 "pièces de percale» pour un bénéficiaire), mais il y a surtout un minimum en dessous duquel le père de la future mariée ne saurait descendre: il ne peut donner moins de 4 "pièces de percale», car un cadeau minime serait indigne: il faut qu'il corresponde au moins à la valeur d'une tête de gros bétail, ne serait-ce qu'un jeune veau.

Bien entendu, l'importance des dons que le père de la future mariée redistribue ainsi, comme le nombre des bénéficiaires de ces dons, dépendent du montant du "prix de la fiancée ». Plus celui-ci est élevé, plus il aura fallu de contributeurs, et plus nombreux à leur tour seront les parents de la mariée qui en recevront une part. Chez les Toubou de 1'Est du Niger par exemple, en 1972, pour un total de 10 mariages, le nombre de parents de la future mariée ayant bénéficié de cette redistribution variait de 5 à 22 , avec une moyenne de 12 personnes par mariage.

Cette large redistribution du " prix de la fiancée » dans la parenté de la mariée est suivie d'une dernière phase de transferts de richesse, qui a lieu le jour même du mariage. La cérémonie se tient dans le campement des 
parents de la future épouse. En zone sahélienne, la date est choisie de préférence à la saison des pluies, car les éleveurs sont alors libérés, grâce à la présence de quelques mares temporaires, des lourdes contraintes de l'abreuvage des troupeaux. A la date arrêtée, la tente nuptiale est d'abord construite. Un grand nombre d'invités sont conviés et diverses réjouissances sont organisées: des danses et des courses de chameaux ou de chevaux ${ }^{6}$. Plus tard, dans la soirée ou la nuit, les parents masculins du garçon et de la jeune fille se réunissent pour " attacher » la partie proprement religieuse du mariage. Ils désignent un tuteur qui interviendra plus tard en cas de difficulté conjugale, et ils fixent le montant de la "garantie du mariage » sadag. Il s'agit d'un don que le mari fait à son épouse selon la règle musulmane. Le plus souvent, ce sont une ou deux têtes de gros bétail, que la femme gardera si par la suite elle est répudiée. Puis les deux groupes de parents, assis en vis à vis, prononcent ensemble la première sourate du Coran, la fatiha, prière qui officialise l'union.

Après ces dispositions religieuses, en général le lendemain en fin d'après-midi, prend place une nouvelle et dernière phase du cycle des transferts matrimoniaux de bétail. Se font donateurs, cette fois, des parents de la mariée. Ils sont ceux-là mêmes qui, dans la phase précédente, avaient bénéficié d'une part de la redistribution faite par le père de la mariée (tewa). Leurs dons, que nous appellerons "animaux du mariage», sont désignés d'un nouveau terme spécifique conofor, pl. conofora. En principe chaque donateur fait un don de valeur équivalente à ce qu'il a reçu, mais il est libre de donner ce qu'il veut, il n'y a pas d'obligation absolue. Par contre, ces dons ne peuvent être que d'une seule nature: il s'agit nécessairement de gros bétail, et ils ont un destinataire unique, le jeune époux.

C'est le père de la mariée qui rassemble, après la grosse chaleur de la mi-journée, ces animaux qu'il a reçus au préalable de ses propres parents et de ceux de sa femme. On frappe le tambour pour appeler tout le monde, puis chaque animal est montré tour à tour, et le nom du donateur ou de la donatrice est énoncé devant la foule des spectateurs qui manifeste bruyamment son approbation: coups de feu, coups de tambour, cris et chants d'allégresse des femmes. Les animaux donnés sont de préférence de jeunes femelles, promesse de croît à venir. Ils sont d'autant plus nombreux que le "prix de la fiancée » était élevé au départ, et la différence est sensible entre mariages riches et pauvres. Ainsi dans une enquête sur 18 mariages échelonnés de 1920 à 1972, menée chez les Toubou de l'Est nigérien, le montant de ces dons variait du simple au triple, avec une moyenne de 18 bêtes par mariage et un maximum de 31 animaux.

\footnotetext{
${ }^{6}$ Le cheval est un animal de prestige réservé à la monte, une sorte de luxe dans ces zones arides.
} 


\section{BILAN DES TRANSFERTS MATRIMONIAUX DE BETAIL}

Si l'on fait le bilan de ces transferts de bétail liés au mariage, on constate qu'ils forment un cycle en quatre étapes, dont chacune implique un nombre important d'animaux. Dans un premier temps, le futur marié fait appel à de nombreux membres de sa parenté pour l'aider à se marier. La richesse qu'il draine ainsi est remise, dans un second temps, à son futur beau-père. Cette richesse, dans un troisième temps, est redistribuée par celui-ci à divers parents de la future mariée. Le nombre de ces bénéficiaires équivaut d'ailleurs plus ou moins à celui des premiers donateurs (phase 1), car le montant du don est voisin dans les deux cas. Ces bénéficiaires, dans un quatrième temps, deviennent donateurs au profit du marié.

L'ensemble de ce processus met en jeu un grand nombre de partenaires. Il aboutit à la formation d'un troupeau entièrement nouveau, composé surtout de jeunes femelles, et c'est ce troupeau qui assurera au couple nouvellement formé la base de son indépendance économique. Ces " animaux du mariage » ont d'ailleurs un statut juridique particulier au sein du troupeau familial : le mari doit gérer ce bétail non pas dans son intérêt personnel, mais dans celui de son épouse et de ses enfants à naître.

C'est seulement après une période de deux ans environ, passés au service de son beau-père, que le jeune marié acquiert son indépendance et peut choisir sa résidence. Il est alors en mesure de récupérer, auprès de son propre père, les animaux qui lui avaient été donnés dans son enfance, à moins que ce dernier n'en ait, entre temps, disposé selon sa convenance. Le troupeau nouvellement constitué, avec lequel le jeune époux débute sa vie de chef de famille autonome, se compose donc de trois catégories juridiques de bétail : 1) les « animaux du mariage » reçus des parents de son épouse le jour du mariage ; 2) les bêtes qu'il a lui-même données à sa femme en " garantie du mariage » lors de la cérémonie ; et 3) celles qui lui avaient été données dans son enfance par divers membres de sa parenté et qu'il peut maintenant récupérer, avec leur croît, dans le troupeau de son père.

Quelle est l'utilité de distinguer ces diverses catégories ? Toutes sont désormais intégrées dans un même troupeau, qui fait l'objet d'une gestion commune. Mais pour autant, les droits exercés sur ces animaux ne sont pas identiques, et les prérogatives du mari, de sa femme et de leurs enfants sur chaque tête de bétail dépendent de la catégorie juridique à laquelle l'animal appartient. De la sorte, les moyens d'action sur le capital familial et les marges de manœuvre dont chacun dispose à cet égard, face à son entourage, dépendent de ces droits. C'est là que ces distinctions juridiques trouvent leur importance : elles impriment leur marque sur les rapports sociaux, et c'est pourquoi il importe de les examiner de plus près. Ceci s'impose d'autant plus que la structuration juridique du troupeau, dans ses grandes 
lignes, reste à peu près stable au fil du temps, en raison d'une règle simple : tout animal à naître appartient, par définition, à la même catégorie que sa mère. C'est d'ailleurs par lignées matrilinéaires que les Toubou décomptent les effectifs de leurs troupeaux ${ }^{7}$. Ils diront par exemple que telle vache « est devenue $\operatorname{dix}^{8} »$ mordom togoso (dix / il (elle) est devenu(e)), telle chamelle « est devenue quinze», etc. Voyons donc en quoi ces catégories juridiques diffèrent, et en quoi les droits qui les caractérisent influent sur les rapports entre les personnes.

\section{LES DIVERSES CATEGORIES JURIDIQUES D'ANIMAUX ET LEUR IMPACT SUR LES RAPPORTS SOCIAUX}

Commençons par le cas le plus simple, celui des animaux qui appartiennent en propre au mari. Ce cheptel peut avoir des origines diverses : dons reçus à la naissance ou à la circoncision, animaux hérités, ou achetés au marché. Les bêtes achetées le sont, le plus souvent, grâce au pécule que certains jeunes Toubou amassent, avant leur mariage, en partant travailler à l'étranger. Certains s'expatrient ainsi, pour un an ou deux, en Libye notamment. Au retour, l'argent gagné leur permet d'acquérir quelques animaux. Après son mariage, le jeune chef de famille répartit d'habitude ses animaux personnels en deux catégories. Il peut allouer une partie de ce bétail à son épouse à titre définitif, et elle dispose alors d'un droit inaliénable au lait de ces bêtes lorsqu'elles sont en lactation. Mais il s'en préserve aussi une autre partie, pour son usage personnel. L'épouse peut alors bénéficier du lait, mais il s'agit d'une situation provisoire et elle ne considérera pas ces animaux comme siens au même titre que les premiers. Son mari, à tout moment, est libre de vendre, donner ou confier une part de ce cheptel à quelqu'un d'autre, sans qu'elle ait son mot à dire sur la question.

Tel n'est pas le cas pour les animaux de la «garantie du mariage ». En volume, c'est souvent la part du troupeau la moins importante, puisqu'il ne s'agit que d'une ou deux bêtes que le mari donne à sa femme le jour de la cérémonie. C'est peu, au regard de la vingtaine d' " animaux du mariage» qu'il reçoit des parents de son épouse. Mais ce n'est pas négligeable pour autant. Tout animal compte aux yeux d'un Toubou d'autant que, la chance aidant, d'un seul animal initial peut résulter un effectif important, au bout de quelques années, si la vache ou la chamelle de départ est très féconde. Les animaux de la « garantie du mariage » appartiennent à la femme, et son mari

\footnotetext{
${ }^{7}$ Comme le font aussi les Peul Wodaabe du Niger (Bonfiglioli, 1988 : 169).

${ }^{8}$ C'est-à-dire qu'en comptant la bête de départ et tous ses petits, on arrive à un total de dix animaux.
} 
ne peut en donner ou en vendre un pour les besoins du ménage sans son accord. S'il se hasarde à le faire sans lui demander son avis, elle est en droit d'exiger restitution de son bien. En cas de répudiation, de plus, l'épouse conserve ce cheptel. De même si son mari décède, ce bétail revient à la veuve et n'est pas décompté dans l'héritage du défunt. Quant au lait de ces animaux, c'est l'épouse qui en bénéficie, à titre exclusif, pour la consommation de son foyer.

Sur la troisième catégorie, celle des "animaux du mariage », les prérogatives de la femme sont moins fortes. Il s'agit d'une part importante du cheptel familial, donnée au mari par les parents de l'épouse. Cette dernière bénéficie, comme pour la catégorie précédente, d'un droit exclusif sur le lait. Son conjoint est le propriétaire de ces animaux, mais ses droits sur eux sont moins étendus que ceux qu'il exerce sur son cheptel personnel. Il a pour mandat de gérer ce bétail dans l'intérêt de sa femme et des enfants qu'elle procrée. Il n'a pas à lui rendre compte de l'usage qu'il en fait; il peut par exemple vendre une bête sans demander l'avis de son épouse ni même l'en avertir. Mais il n'est pas totalement libre pour autant de faire entièrement ce qu'il veut. Par exemple, il est hors de question qu'il dilapide ce bien à des fins personnelles, pour favoriser abusivement ses parents et encore moins pour acquérir une seconde épouse. Dans un tel cas, la famille de la première serait en droit d'intervenir pour protéger les intérêts de leur parente ainsi abusée. D'ailleurs si le mari décède sans descendance, ces animaux ne reviennent pas aux parents du défunt comme c'est le cas de son bétail personnel : un quart des « animaux du mariage» est conservé par sa veuve et le reste est restitué aux donateurs, parents de cette dernière.

Par le biais du bétail ainsi que par leur ascendance morale, les membres de la famille d'origine de l'épouse jouent d'ailleurs, dans le devenir du couple, un rôle qui est loin d'être négligeable. En cas de mésentente conjugale notamment, le conflit revient généralement entre leurs mains car l'épouse mécontente prend la fuite. Elle revient chez son père, et c'est à lui et à ses proches d'arbitrer le différend. Il peut inciter sa fille à retourner chez son mari, ce qui est un cas fréquent. Mais s'il juge que le mari est dans son tort, il lui demande réparation et ne lui rend son épouse qu'en échange d'une tête de gros bétail. Ce processus se répète parfois, et les querelles de ménage peuvent alors en coûter cher au mari. Lorsqu'elles persistent, le beau-père peut en venir à considérer la mésentente du couple comme rédhibitoire, et refuser de rendre sa fille à son époux. Par son rôle d'arbitre en cas de difficultés conjugales, le père d'une femme mariée lui apporte donc un soutien essentiel dans sa vie de couple.

Par le biais du bétail également, le père et les autres membres de la parenté de l'épouse jouent sur son foyer un rôle crucial, qui se prolonge 
longtemps après le mariage. En effet, les « animaux du mariage » qu'ils ont donnés à son mari le jour de la cérémonie peuvent n'être que l'amorce de dons beaucoup plus importants. Ceux-ci se font au fil du temps et souvent de nombreuses années plus tard. Ces dons, bien entendu, dépendent de la richesse de ces personnes, et c'est pourquoi il est particulièrement désirable, aux yeux des Toubou, d'épouser une femme dont les parents sont riches et nombreux. Mais ces dons postérieurs au mariage dépendent aussi de la considération que les parents de l'épouse ont pour leur gendre. Plus leur estime est forte, plus ils se montreront généreux. L'épouse elle-même, d'ailleurs, peut les inciter à cette générosité en faisant une tournée de visites dans sa parenté, par exemple après la naissance d'un enfant. Beaucoup de femmes font cette démarche une fois dans leur vie. Elles emportent du thé, $\mathrm{du}$ sucre, du mil et quelques menus cadeaux pour leurs parents, et reviennent au bout de quelques semaines ou même d'un mois ou deux, avec plusieurs animaux. Leur nombre peut atteindre une quinzaine ou davantage (Baroin, 1985 : 253-256). Ce bétail intègre la catégorie des « animaux du mariage » donnés lors des noces, ils sont sujets aux mêmes droits. Mais ces dons ne sont pas sans retour, car les liens d'alliance ainsi renforcés sont aussi des liens de solidarité. En particulier, lorsqu'un jeune membre de la parenté de l'épouse cherchera par la suite à se marier, il est bien naturel qu'il vienne solliciter cette parente et son conjoint pour lui donner un animal afin de rassembler le «prix de la fiancée ». D'ailleurs, c'est précisément dans cette catégorie des «animaux du mariage» que le don sera prélevé.

La façon dont les parents de l'épouse peuvent ainsi peser sur l'avenir $\mathrm{du}$ couple, tant du point de vue moral qu'économique en accroissant le capital des « animaux du mariage », module fortement les rapports du mari envers ses parents par alliance, ainsi que les rapports des conjoints entre eux. Le mari, à bien des égards, est l'obligé de ses beaux-parents et il a tout intérêt à ménager l'opinion qu'ils ont de lui, car c'est d'elle que dépendent leurs largesses. Quant à sa femme, elle dispose grâce au poids de sa parenté d'un moyen de pression efficace dans ses rapports de couple. L'influence de ses parents l'autorise à une certaine liberté de comportement face à son mari. Ce n'est pas, loin s'en faut, une liberté de mœurs mais l'indépendance de ton et la fierté des femmes toubou surprend nombre d'observateurs non avertis. Les femmes toubou sont subordonnées à leurs maris, certes, mais ce ne sont pas des femmes soumises car chacune sait qu'elle peut, en cas de difficulté, compter sur le soutien de sa famille d'origine qui interviendra pour défendre ses droits et auprès de laquelle, en ultime instance, elle pourra trouver refuge. La solidarité des parents par le sang est un principe qui va de soi, alors que le mariage peut être précaire surtout s'il n'y a pas d'enfant. 
Aussi est-ce le nombre de ses enfants, et surtout de ses fils, qui renforce une femme dans sa position d'épouse. Leur présence éloigne le risque de répudiation, d'autant qu'il serait disgracieux pour un mari de répudier une femme dont il a eu plusieurs enfants.

Le principe absolu de la solidarité entre parents par le sang motive, de façon plus générale, l'attitude des femmes en matière de propriété du bétail. Que se passe-t-il, en effet, lors d'un partage d'héritage ? Comme les Toubou sont musulmans, les fils selon la règle héritent double des filles. La part féminine est donc réduite, mais il arrive néanmoins qu'une fille reçoive en héritage, au décès de son père, un grand nombre d'animaux. Tel est le cas si le défunt n'a laissé aucun fils, ou s'il était très fortuné. Sa ou ses filles se voient alors attribuer un important cheptel. Mais ces riches héritières, plutôt que d'intégrer ce bétail dans le troupeau de leur conjoint, préfèrent souvent renoncer à leur part au profit de leurs frères ou oncles, ou à tout le moins leur en laisser la garde. Ce choix est bien le signe qu'elles ont une confiance plus forte en leurs proches parents qu'en leur conjoint. Quand leurs fils sont adultes par contre, c'est à eux que les femmes d'âge mûr abandonnent les animaux qui leur restent, d'autant que c'est toujours aux fils en priorité d'assumer la charge de leur mère vieillissante.

Les partages d'héritage introduisent dans le cheptel familial des animaux nouveaux, d'un statut différent des précédents. Ceci modifie la composition juridique du troupeau, ainsi que la proportion de chaque catégorie dans l'effectif global. Le bétail reçu en héritage par le mari s'ajoute simplement à son cheptel personnel et ne forme donc pas de catégorie nouvelle. Par contre l'héritage de l'épouse, quand elle le confie à son conjoint, forme dans le troupeau familial une catégorie distincte, qui ne s'assimile ni à la "garantie du mariage », ni aux « animaux du mariage». Elle seule détient les droits sur ces animaux. S'ils forment une proportion importante du troupeau familial, l'autorité de la femme face à son mari s'en trouve accrue. Nous avons eu l'occasion, notamment, d'observer un ménage dont l'épouse était considérablement plus riche que son conjoint. Cette situation, peu fréquente sans être exceptionnelle, se traduisait en particulier dans la résidence de ce couple. En effet, l'emplacement de la tente familiale est normalement choisi par le mari, qui opte fréquemment pour le voisinage de ses frères ou autres proches parents. Mais la tente de cette femme fortunée, contrairement à l'habitude, était montée au voisinage de ses propres parents, et non de ceux de son mari.

Un partage d'héritage peut aussi grossir le troupeau d'un ménage par un autre biais. Car ce ne sont pas seulement les animaux hérités par le mari ou la femme qui peuvent s'y ajouter, mais aussi, le cas échéant, ceux d'une sœur du mari ou d'une autre de ses parentes proches. Cette dernière aura 
choisi de confier ce bétail hérité à son parent, plutôt que de le garder avec elle, comme nous l'avons vu ci-dessus. Le troupeau familial comporte alors des animaux qui n'appartiennent ni au mari ni à son épouse, et cette situation est d'autant plus fréquente que cette forme de prêt de bétail ne résulte pas seulement de l'héritage. Un riche éleveur, par exemple, peut choisir de confier une partie de ses animaux à un parent moins fortuné. C'est une manière de lui venir en aide, et c'est aussi une stratégie de diversification des risques. Le bétail confié ne change pas de propriétaire, il est simplement géré par quelqu'un d'autre.

Rappelons aussi qu'à mesure que naissent les enfants, le troupeau familial se grossit des dons d'animaux faits aux bébés, puis aux garçons lors de leur circoncision, par des parents paternels ou maternels. Ce bétail reste propriété nominale de l'enfant bénéficiaire, mais le père de famille, comme nous l'avons vu, le gère à sa convenance jusqu'au mariage de l'intéressé. Si les nécessités du ménage l'y poussent, il peut vendre une partie de ce cheptel ou de sa descendance.

Au total, il est donc bien clair que le troupeau familial, du point de vue juridique, ne forme pas, loin s'en faut, un tout indifférencié. Il est composé de catégories juridiques multiples, formée chacune d'un nombre plus ou moins grand d'animaux. Cette composition dessine une structure juridique globale du troupeau, qui conditionne les marges de manœuvre du père de famille dans sa gestion de ce cheptel. Ce profil, toutefois, n'est pas rigoureusement identique dans chaque cas. Les troupeaux diffèrent par leur taille, mais aussi par la proportion des diverses catégories de bétail qui les composent. Dès le départ, les situations sont variables puisque le nombre des « animaux du mariage », notamment, peut être plus ou moins important par rapport au cheptel personnel du mari. De plus, la structure initiale évolue au fil du temps. Elle est modulée d'un côté par des apports, et de l'autre par des ponctions qui affectent de manière différenciée chaque catégorie, qu'il s'agisse de dons, d'héritage, de prêts dans un sens ou dans l'autre. Le profil juridique du troupeau dépend aussi d'un autre facteur, qui est le hasard. La chance ou divers avatars peuvent affecter une catégorie plus qu'une autre, certains animaux s'avérant plus féconds que d'autres, tandis qu'une lignée animale peut être sujette, plus qu'une autre, à la mort ou à la maladie. Grande est donc la variabilité des situations, mais il n'en reste pas moins que c'est dans la même grille juridique que s'inscrit, avec ses variantes, la structure de chaque troupeau. Cette dernière, loin d'être un fait anodin, mérite pleinement l'attention car elle éclaire, sous un jour essentiel, bien des comportements conjugaux ou parentaux.

La structure juridique des troupeaux résulte dans une large mesure des réseaux d'échanges matrimoniaux précédemment décrits. A travers elle, se 
joue l'impact de ces réseaux sur les rapports sociaux, que ce soient les rapports de parenté et d'alliance, les rapports conjugaux ou le statut de l'épouse. Mais au-delà des relations entre les individus, ces réseaux d'échanges ont aussi d'autres conséquences à l'échelle de la société globale, dans les domaines économique et politique.

\section{ECHANGES ET VIE ECONOMIQUE}

Du point de vue économique, pour chaque nouveau mariage le système matrimonial toubou impulse un cycle d'échanges auquel participent un grand nombre de cellules familiales, apparentées les unes au futur marié, les autres à son épouse. Le mariage constitue donc un puissant moteur des échanges au sein de la société toubou. Ceux-ci se prolongent de surcroît pour chaque union, au fil de la vie maritale du couple. Ils instaurent entre parentèles alliées des relations d'entraide réciproques et durables.

Mais il s'ajoute, à la circulation de bétail liée au mariage, d'autres motifs de transferts d'animaux. Nous avons évoqué plus haut les dons faits à la naissance et à la circoncision, ainsi que l'héritage. Il faut y ajouter les compensations versées à une victime ou à ses proches en cas de dommages corporels ou de meurtres. Leur importance est loin d'égaler les échanges matrimoniaux, mais ces versements, pour autant, ne sont pas rares en pays toubou. Un meurtre ou un préjudice corporel entraîne une obligation de vengeance pour les parents de la victime, à laquelle seul peut mettre un terme le versement d'une compensation en bétail par les parents de l'agresseur aux parents de la victime. Un barème est prévu, selon l'importance du dommage, qui suit en principe le rite malékite dont relèvent les Toubou, stipulé par La Risâla (Baroin, 1985 : 163). Pour un meurtre, cette compensation se monte, chez les Toubou du Niger, en principe à 100 vaches pour un homme, 50 pour une femme, indemnités qui sont conformes, dans leurs proportions, aux préceptes de l'islam.

L'obligation pour les parents proches de contribuer à ces compensations pour meurtre ou blessure, comme aux paiements de mariage, est telle que tout éleveur, bien que formellement autonome, ne peut considérer son cheptel comme lui étant définitivement acquis. En effet, il peut à tout moment devoir se défaire d'un ou plusieurs animaux pour répondre à ses obligations sociales. Au bout du compte, la vie économique des Toubou se caractérise donc par une circulation intense de bétail puisque des transferts innombrables d'animaux se produisent, d'une cellule familiale vers une autre, en des occasions multiples et renouvelées qui s'égrènent au fil du temps, et parmi lesquelles les paiements et les dons de mariage jouent un rôle primordial. 
Ces échanges s'effectuent tous au sein même de la société toubou. Ils n'ont qu'un rapport anecdotique avec l'économie de marché, lorsqu'un futur marié va vendre le bétail qu'il a reçu de ses parents pour acheter du thé et du sucre, si tel est le type de versement que requiert son beau père. Ceci infirme, bien sûr, l'idée trop souvent admise qu'il ne saurait y avoir d'économie à proprement parler en dehors de l'économie mercantile.

\section{LA VIE POLITIQUE : ECHANGES ET ANARCHIE}

Cette sociabilité en réseaux n'influe pas seulement sur la vie économique de ces pasteurs. Ses conséquences se font aussi sentir dans le domaine politique. En effet chaque famille restreinte est insérée dans un vaste entrecroisement de relations d'entraide, impliquant consanguins et alliés, de sorte qu'elle est à la fois autonome et solidaire des autres. Il en résulte un maillage social fluide, sans centre ni périphérie, où chaque Toubou se situe au centre d'un réseau qui lui est propre. Cette forme d'organisation permet à chacun de se sentir indépendant et de considérer qu'il n'a de comptes à rendre à personne.

Ce sentiment correspond d'ailleurs à une réalité car s'il existe bien des chefs chez les Toubou (de lignages ou de clans), ceux-ci n'ont tout au plus qu'un rôle d'arbitre. Ils n'ont aucun pouvoir coercitif sur les membres de leur groupe patrilinéaire. Les membres d'un clan, ou même d'un lignage, sont très disséminés géographiquement et ne se réunissent jamais. Le clan toubou ne constitue donc pas un "groupe en corps » corporate group. Il s'agit plutôt d'une sorte de blason honorifique, défini par plusieurs attributs : une tradition historique, un surnom, un interdit et une marque de clan (Baroin, 1985 : 83-92). Il appartient aux membres d'un même clan de veiller à ce que soient respectés ces insignes communs de leur honneur, en réagissant aux éventuelles insultes dont seraient l'objet leur interdit ou leur surnom, en vengeant un meurtre ou en poursuivant le voleur d'animaux portant les marques de bétail du clan. De ce fait, le rôle des clans toubou s'exerce surtout dans le domaine de la régulation de la violence, en particulier lorsqu'il y a meurtre ou vol de bétail. Or ce sont des occasions fréquentes dans cette société traditionnellement guerrière, où le vol de bétail est valorisé comme une marque d'audace. Mais dans de tels cas, la solidarité se limite néanmoins aux parents les plus proches car "les obligations de vengeance [...] ne dépassent pas [...] un certain degré de parenté à l'intérieur du clan" (Chapelle, $1957:$ 346).

En matière politique, on peut donc dire que le clan toubou, qui ne correspond pas aux réalités sociologiques auxquelles réfère habituellement ce terme, alimente des obligations de vengeance et la violence endémique de cette société, comme sa régulation. L'esprit d'indépendance des Toubou, 
l'absence chez eux de "groupes en corps » et de chefferies fortes contribuent à cette anarchie, au sens étymologique du terme, qui caractérise leur société. On conçoit d'ailleurs mal, dans cette logique sociale à base de réseaux personnels entremêlés, comment une chefferie forte pourrait trouver sa place.

\section{BILAN COMPARATIF}

Il est instructif de comparer ce système social à celui des quelques sociétés pastorales où la circulation et les droits sur le bétail ont été étudiés. Nous disposons à cet égard des travaux pionniers de Gulliver (1955) sur les Jie d'Ouganda et les Turkana du Kenya, de ceux de Dupire (1962) et Bonfiglioli (1988) sur les Peul Wodaabe du Niger, et de ceux d'Almagor (1978) sur les Dassanetch d'Ethiopie. Esquissons donc quelques brèves comparaisons.

Il existe, entre les Toubou et les Peuls Wodaabe du Niger, des similitudes frappantes. Dans les deux cas la chefferie est faible et la société se compose de petites unités socio-économiques indépendantes, liées par des rapports personnels à un vaste réseau de relations. Nombre de remarques essentielles de Bonfiglioli sur les Wodaabe valent aussi bien pour les Toubou :

La tendance générale de la société bodaado [...] est celle d'une constitution rapide d'unités sociales et économiques indépendantes [...] Cependant, cette tendance vers la constitution d'unités petites, indépendantes et flexibles ne signifie pas que ces unités peuvent vivre isolées les unes des autres : elles doivent toujours être situées dans le cadre d'une structure d'appoint plus vaste, de manière que les risques soient partagés et que les unités individuelles puissent s'appuyer sur les autres en cas de besoin. Ainsi, chez les Wodaabe, comme dans la plupart des autres sociétés pastorales, une unité familiale ne constitue pas un isolat. Chaque unité familiale / unité de production fait partie d'une structure sociale complexe, d'un véritable réseau de relations. Entre les différentes unités familiales, l'aspect le plus visible de ces liens sociaux et pastoraux est l'intense circulation de personnes et d'animaux entre les unités : les personnes, à cause des mariages, des voyages, des séjours plus ou moins prolongés ; les animaux, à cause de tous les systèmes de dons, prêts, échanges, allocations et transferts.

(Bonfiglioli, 1988 : 163-165).

Cet auteur remarque également (p. 165) qu'un troupeau "ne constitue pas une masse homogène, mais un ensemble hétérogène de catégories d'animaux", comme l'avait montré avant lui Dupire (1962: 114-123). Toutefois, ces catégories hétérogènes diffèrent dans le cas des Peuls Wodaabe et des Toubou, en raison notamment de règles matrimoniales diamétralement opposées. Alors que l'interdiction de mariage entre parents est très étendue chez les Toubou, c'est au contraire le mariage entre enfants de frères qui est préféré chez les Peuls Wodaabe, au point que la fréquence de ces mariages "supprime la notion d'échange" (Dupire, 1962 : 241). A 
l'inverse, l'alliance entre parentèles étrangères renforce chez les Toubou la nécessité de l'échange au point que celui-ci en devienne la clé de voûte de leur économie et de leur architecture sociale, en donnant lieu notamment à la création d'une catégorie juridique très importante dans les troupeaux, celle des conofora. Rien de tel dans les troupeaux wodaabe, répartis pour l'essentiel en trois catégories: a) les animaux provenant du troupeau paternel, b) ceux qui viennent du cheptel attribué au départ par le père à la mère, et c) les "vaches d'attache " ( $c f$. ci-après l'article de Jean Boutrais) qui résultent de prêts de divers parents et amis (Bonfiglioli, 1988: 154). Dans l'exemple fourni, les deux premières catégories, qui sont d'origine agnatique de manière directe ou indirecte, constituent $70 \%$ du troupeau. Le poids du groupe agnatique est donc beaucoup plus marqué chez les Peuls Wodaabe que chez les Toubou, aussi bien dans la détermination des mariages que dans la formation des troupeaux. Cette différence se marque aussi dans le domaine politique, puisque ni les clans ni les lignages toubou ne sont des unités géographiques alors que la fraction agnatique wodaabe, qui réunit au maximum une cinquantaine de campements, conduite par son chef, forme "une unité de nomadisation pouvant se déplacer saisonnièrement et émigrer seule au gré des circonstances" (Dupire 1962 : 289).

Chez les Dassanetch de la basse vallée de l'Omo, dans le Sud éthiopien, la question de la circulation et des droits sur les animaux a été étudiée en détail par Almagor (1978). Ces agro-pasteurs sont organisés en unités familiales autonomes et néanmoins reliées à de vastes réseaux interpersonnels d'entraide, comme les Wodaabe et les Toubou. Toutefois leur vie politique et rituelle diffère: elle est régulée par un système de classes d'âge et de générations complexe et centralisé, qui entraîne aussi d'importantes restrictions matrimoniales, comme c'est le cas de nombreuses sociétés pastorales ou agro-pastorales d'Afrique de l'Est. Par ailleurs, la vie sociale des Dassanetch est alimentée par une circulation intense de bétail entre les familles.

Ces agro-pasteurs vivent dans un environnement naturel très propice, mais géographiquement limité par la présence d'ethnies voisines. Ils évitent la surpopulation animale grâce à un abattage massif d'animaux, consommés lors des rituels, alors que chez les Toubou et les Wodaabe au contraire, l'espace disponible est plus vaste et l'objectif des éleveurs est la maximisation des troupeaux.

La compensation matrimoniale des Dassanetch peut atteindre jusqu'à 80 animaux (Almagor, $1978: 174$ ), alors qu'elle est insignifiante chez les Wodaabe et se monte de 10 à 25 têtes de gros bétail chez les Toubou (cf. cidessus). Mais surtout, elle n'est pas fixée au départ et ne se verse pas avant le mariage comme chez les Toubou, mais au fil des vingt à trente années qui 
suivent la naissance du premier enfant (ibid. : 10 et 66). Ces versements, de plus, sont la cause de nombreuses disputes, les ayant droit exerçant une pression constante sur l'époux pour obtenir leur dû (ibid.: 181). Autre différence, les droits non versés s'héritent à la génération suivante et peuvent occasionner des sanctions surnaturelles en cas de non paiement (ibid. : 183). A l'inverse des Toubou, le père de l'épouse ne reçoit aucun de ces animaux ; ils sont donnés directement au grand nombre d'autres parents, alliés ou partenaires d'échange de ce dernier.

Les Dassanetch complètent l'éventail des obligations entre parents et alliés par quatre types de "partenariats spécifiques » bond partnerships institués par des rituels, qui sont exposés en détail par Almagor dans son chapitre V. Ces liens entraînent des obligations d'assistance, des interdictions de mariage et des droits sur les compensations matrimoniales des filles des partenaires. Pour le plus fort de ces contrats, celui qui unit le père d'un nouveau né à l'homme dont le bébé portera le nom, les droits sur les compensations matrimoniales s'étendent aux sœurs et aux nièces des partenaires, lesquels sont considérés comme des frères (ibid. : 119-121).

Almagor souligne combien, dans cette société fortement égalitaire, le poids d'un homme se mesure à la quantité et à la qualité de ses liens sociaux, qui fluctuent en permanence et ne débouchent sur aucun pouvoir durable du fait qu'ils ne sont pas transmis à la génération suivante (ibid. : 10-11 et 248-249). Bonfiglioli (1988: 185), de même, remarque que la richesse est fluctuante et aléatoire aux yeux des Peul Wodaabe, ce qui "conduit à un type de société qui se construit sur des bases égalitaires, tout au moins dans le sens que la propriété d'un troupeau ne constitue pas en soi la base d'une stratification sociale". Chez les Toubou tout autant, la stratification sociale est inexistante. Leur littérature orale témoigne même d'une défiance unanime envers les autorités constituées, qui participe d'une "véritable culture de l'anarchie" (Boutrais", 2007 : 39-40).

Envisageons pour finir le cas des Jie d'Ouganda et des Turkana du Kenya, peuples qui relèvent du cercle Karimojong ${ }^{10}$ et dont l'origine est commune. Gulliver leur a consacré en 1955 un ouvrage comparatif, Family Herds, qui était novateur en son temps car en dépit de la multiplicité des études sur les sociétés pastorales, peu de chose avait été publié jusque là sur la sociologie de la propriété du bétail (ibid. : 1-2). Les Jie sont des agro-

\footnotetext{
${ }^{9}$ Boutrais, Jean, 2007, compte rendu de Tubiana, J., (éd.), 2007. Contes toubou du Sahara ; quatre-vingt-dix-neuf contes, mythes et chansons, Paris, L'Harmattan, 199 p. in Méga-Tchad, pp. 38-40.

${ }^{10}$ Le « cercle Karimojong » Karimojong cluster est un groupe de populations culturellement apparentées, qui comprend aussi en Ouganda les Karimojong, les Teso, les Dodoth et les Labwor, au Soudan les Toposa et les Jiye, ainsi que les Nyangatom du sud de l'Ethiopie (Gulliver, 1952 ; Tornay, $2001: 80-81$ ).
} 
pasteurs, tandis que les Turkana sont des éleveurs qui nomadisent en zone aride à l'est du lac Turkana. Les uns comme les autres n'ont ni chef ni stratification sociale : "there is no controlling authority to override personal choice and inclination" (ibid. : 32). Ils n'ont pas non plus de "groupes en corps » au delà de la famille étendue pour les premiers, de la famille restreinte pour les seconds. Gulliver souligne l'importance de la compensation matrimoniale chez les Jie et les Turkana: elle se monte en moyenne à 50 têtes de gros bétail et jusqu'à 200 chèvres ou moutons, le nombre des contributeurs pouvant aller jusqu'à 50 ou plus (ibid. : 197). La circulation de bétail qui en résulte est d'autant plus importante que la polygynie est fréquente dans les deux cas (ibid. : 242-243) ${ }^{11}$. La moitié de la compensation est prise dans le troupeau personnel du futur mari, tandis que l'autre lui est donnée par ses «partenaires de bétail» stock associates (ibid.: 197). Le nombre de ceux-ci dépend de l'âge et de la richesse de chacun; il peut varier de 7 à 8 jusqu'à une cinquantaine, avec une moyenne de 30 associés environ par personne (ibid.:215). Ces «partenaires de bétail » comprennent les parents cognatiques et parents par alliance proches, ainsi que des «amis» bond-friends auxquels chacun se lie par «engagement délibéré» deliberate pledge (ibid.:196-198) mais sans cérémonie particulière (ibid.: 210), au contraire des Dassanetch. Gulliver souligne combien ces partenariats de bétail sont au cœur de la vie sociale car bien qu'il n'y ait pas de réciprocité rigoureuse (ibid. : 221), ils entraînent des droits et devoirs réciproques d'hospitalité, d'assistance, de soutien en cas de conflit, de dons d'animaux pour reconstituer un troupeau ou de nourriture en cas de besoin, d'aide en main d'œuvre, en droits d'accès aux points d'eau ainsi que le don spontané sans raison spécifique, le prêt et le confiage mutuel de bétail (pp. 199-200). Ainsi se tisse une sociabilité en réseaux sur la base de liens entre partenaires associés un à un, chacun ayant son propre cercle, de façon tout à fait comparable à ce qui s'observe chez les Toubou, les Peul Wodaabe et les Dassanetch.

Il y a donc, entre ces sociétés que nous venons d'évoquer, des similitudes remarquables. On y observe la même circulation intense de bétail entre des cellules familiales qui sont liées entre elles par de multiples droits et obligations interindividuels. Ces droits et obligations, distincts dans chaque cas, proviennent soit de liens de parenté, soit de liens d'alliance, soit encore de partenariats entre amis dont la formalisation est plus ou moins poussée : simple amitié chez les Jie, les Turkana et les Toubou, «vache d'attache » chez les Peul Wodaabe, "partenariats spécifiques » institués par des rituels particuliers chez les Dassanetch. Les droits sur le bétail, à

${ }^{11}$ Il en est de même chez les Dassanetch (Almagor, 1978 : 167). 
l'intérieur d'un troupeau, diffèrent selon les catégories en cause, lesquelles dépendent de l'origine de chaque animal. Mais autant que cet éventail des droits de propriété qui s'exercent sur le cheptel composant, à un moment donné, le troupeau d'une famille, ce qui compte c'est la dynamique de la constitution de ce cheptel. Sa composition est éminemment variable, au fil du temps, car elle ne dépend pas seulement de la fécondité des bêtes ou des aléas d'une sécheresse, elle dépend aussi, à la fois de l'obligation de donner une partie de ces animaux à divers partenaires en fonction de circonstances précises, et du droit à recevoir d'autres bêtes d'autres personnes, pour d'autres raisons. Nous ne pouvons donc que faire écho à Gulliver en insistant sur l'intérêt d'étudier cette sociologie de la propriété du bétail. Elle est au cœur de la vie sociale des pasteurs, comme elle est le moteur de leur vie économique.

Quant au niveau politique, on peut faire à son propos plusieurs remarques. Il existe, certes, des différences notables entre ces sociétés et en particulier, l'organisation en classes d'âge et de génération qui s'observe en Afrique de l'Est - chez les Dassanetch, les Jie et les Turkana - est absente chez les pasteurs d'Afrique occidentale, notamment chez les Peul Wodaabe et les Toubou. Néanmoins, on constate certaines convergences dans la conception de la richesse : celle-ci tient tout autant à l'ampleur du réseau social de chaque individu qu'au nombre d'animaux qu'il possède. Dans chacune de ces sociétés, de fortes solidarités interindividuelles vont de pair avec une tendance à l'égalitarisme, que les Toubou poussent même jusqu'à un véritable esprit d'anarchie. Il ne reste donc plus qu'à espérer que d'autres études sur ce thème viennent enrichir ces quelques réflexions.

\section{Références bibliographiques}

Almagor, U., 1978, Pastoral partners. Affinity and bond partnership among the Dassanetch of South-West Ethiopia, Manchester, Manchester University Press, $258 \mathrm{p}$.

BAROIN, C., 1985, Anarchie et cohésion sociale chez les Toubou : les Daza Kécherda (Niger), Cambridge, Cambridge University Press ; Paris, Ed. de la $\mathrm{MSH}$, col. Production pastorale et société, $456 \mathrm{p}$.

-, (éd.), 1988, Gens du roc et du sable - Les Toubou. Hommage à Charles et Marguerite Le Cour, textes réunis par Catherine Baroin, Paris, Ed. du C.N.R.S., 286 p.

- 1994, Querelles et droits fonciers au Borkou, Droit et cultures, 28, pp. 119141.

—, 2005, Pudeur et nourriture : les manières de table des Toubou, in Raimond C., E. Garine et O. Langlois (éds), Ressources vivrières et choix alimentaires dans le bassin du lac Tchad, Paris, IRD, pp. 377-395. 
Bonfiglioli, A. M., 1988, Dudal. Histoire de famille et histoire de troupeau chez un groupe de Wodaabe du Niger, Cambridge, Cambridge University Press ; Paris, Ed. MSH, col. Production pastorale et société, 293 p.

Dupire, M., 1962, Peuls nomades. Etude descriptive des Wodaabe du Sahel nigérien, Paris, Institut d'ethnologie, $338 \mathrm{p}$.

Gulliver, P. H., 1952, The Karamojong Cluster, Africa, 22, 1, pp. 1-22.

-, 1955, The Family Herds. A study of two pastoral tribes in East Africa, the Jie and Turkana, Londres, Routledge and Kegan Paul, 273 p.

La Risâla. Epitre sur les éléments du dogme et de la loi de l'Islam selon le rite mâlikite, par Ibn Ab̂̀ Zayd Al-Qayrawân̂, traduction française par Léon Bercher. Alger, Editions Populaires de l'Armée, $6^{\text {ème }}$ éd. 1975.

SwIFT, J., 1979. The Development of Livestock Trading in a Nomad Pastoral Economy: The Somali Case, in Equipe écologie et anthropologie des sociétés pastorales (éd.), Production pastorale et société, Cambridge, Cambridge University Press, Paris, Ed. MSH, col. Production pastorale et société, pp. 447-465.

TORNAY, S., 2001, Les fusils jaunes. Génération et politique en pays nyangatom (Ethiopie), Nanterre, Société d'ethnologie, 363 p. 